\title{
ONREL
}

\section{"The Durability of Polymeric Encapsulation Materials for Concentrating Photovoltaic Systems"}

David C. Miller ${ }^{1 *}$, Matt Muller ${ }^{1}$, Michael D. Kempe', Kenji Araki', Cheryl E. Kenned ${ }^{1}$, and Sarah R. Kurtz ${ }^{1}$

1. National Renewable Energy Laboratory (NREL), 15013 Denver West Parkway, Golden, CO, USA 80401

2. Daido Steel Co., Ltd. 2-30 Daido-cho, Minami, Nagoya 457-8545, Japan

* David.Miller@nrel.gov

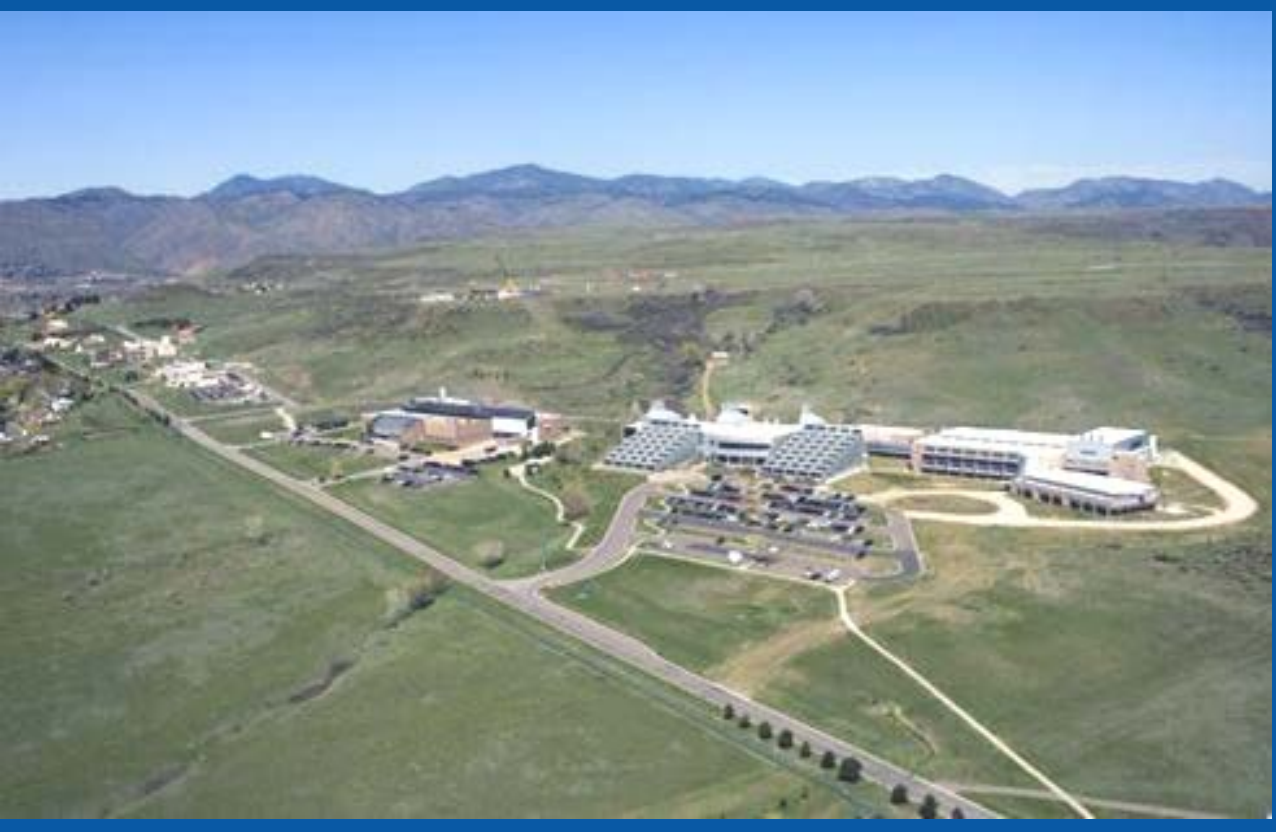

2012 PV Module Reliability Workshop

(Denver West Marriot, Golden, CO)

8:30-8:50 am, 2012/3/01 (Thursday)

Golden Ballroom

-this presentation contains no proprietary information- 


\section{Motivation for the NREL Field Study}

- Concentrating Photovoltaic (CPV) modules use cost effective optics (\$) to focus light onto high efficiency $(\eta=44 \%)$ multijunction cells ( $\$ \$ \$ \$$ )

\section{solarflux}

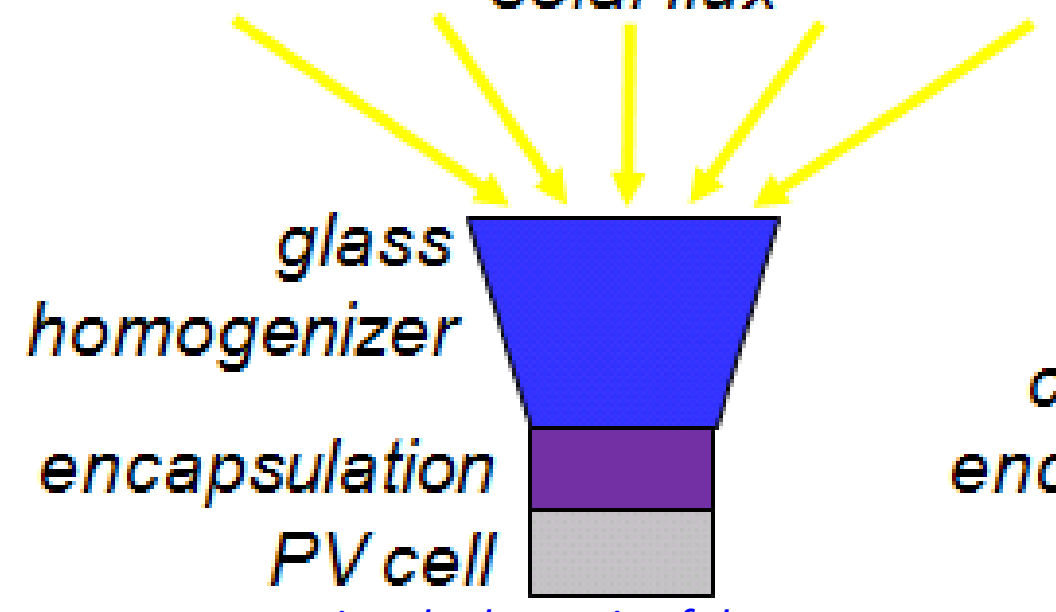

coverglass encapsulation PV cell

solarflux

cross-sectional schematic of the components near the cell in CPV systems (not to scale) 


\section{Motivation for the NREL Field Study}

- Concentrating Photovoltaic (CPV) modules use cost effective optics (\$) to focus light onto high efficiency $(\eta=44 \%)$ multijunction cells ( $\$ \$ \$ \$$ )

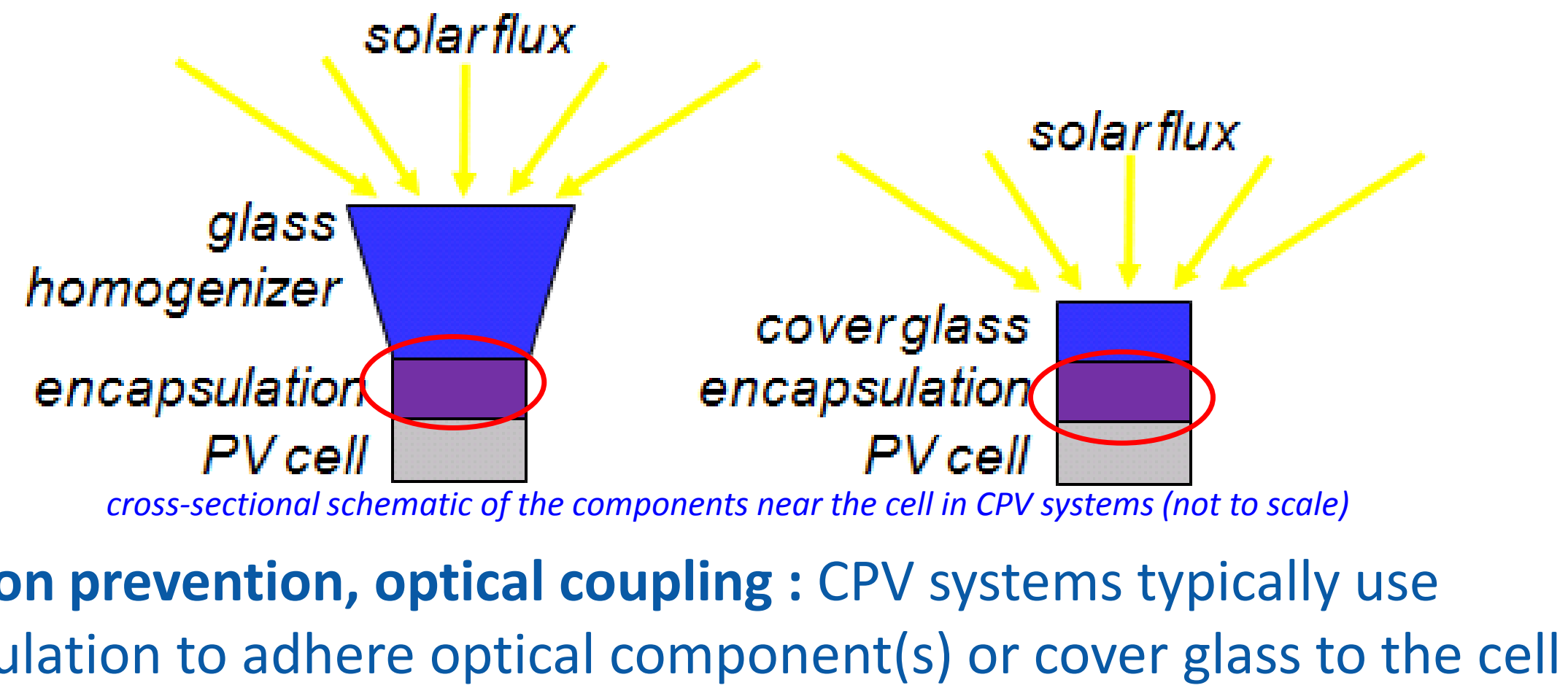




\section{Motivation for the NREL Field Study}

- Concentrating Photovoltaic (CPV) modules use cost effective optics (\$) to focus light onto high efficiency $(\eta=44 \%)$ multijunction cells $(\$ \$ \$ \$$ )

\section{solarflux}

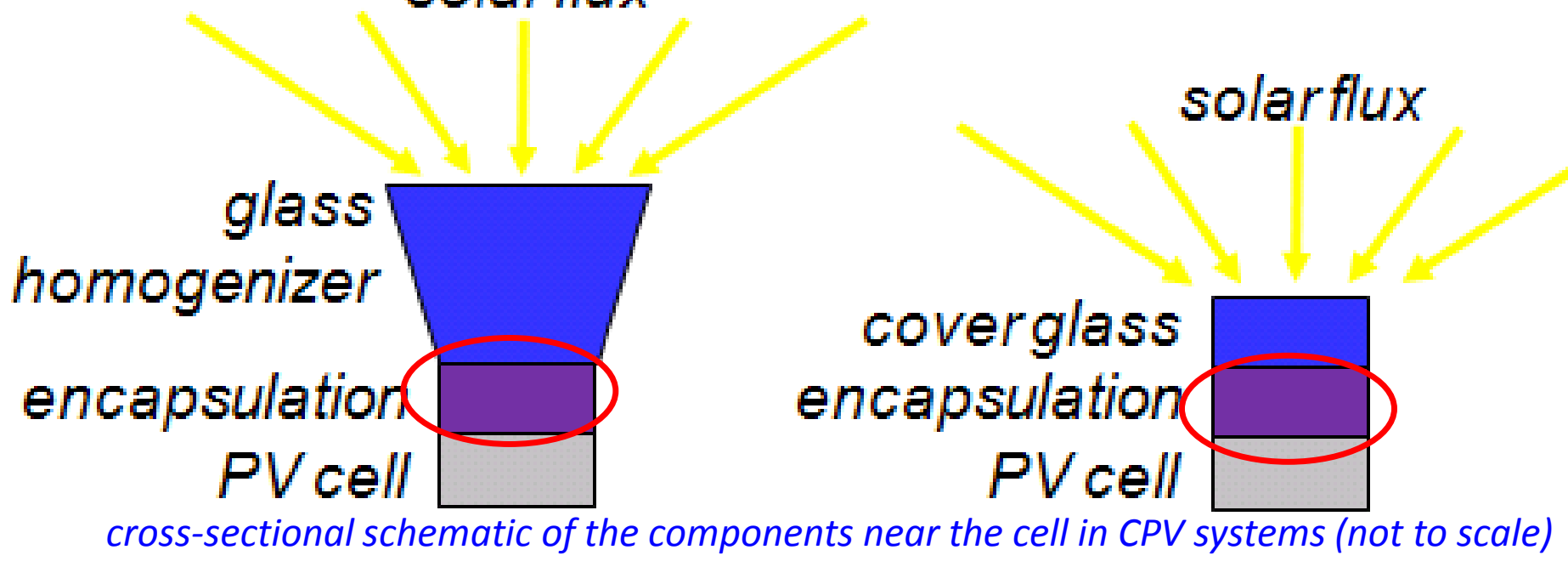

corrosion prevention, optical coupling : CPV systems typically use encapsulation to adhere optical component(s) or cover glass to the cell encapsulation durability ( 30 year field deployment) is unknown: -identify field failure modes

-gain insight related to failure mechanisms

- distinguish between material types

-identify materials for future study (HALT \& qualification tests) 
Miller et. al., PIP, DOI: 10.1002/pip.1241.

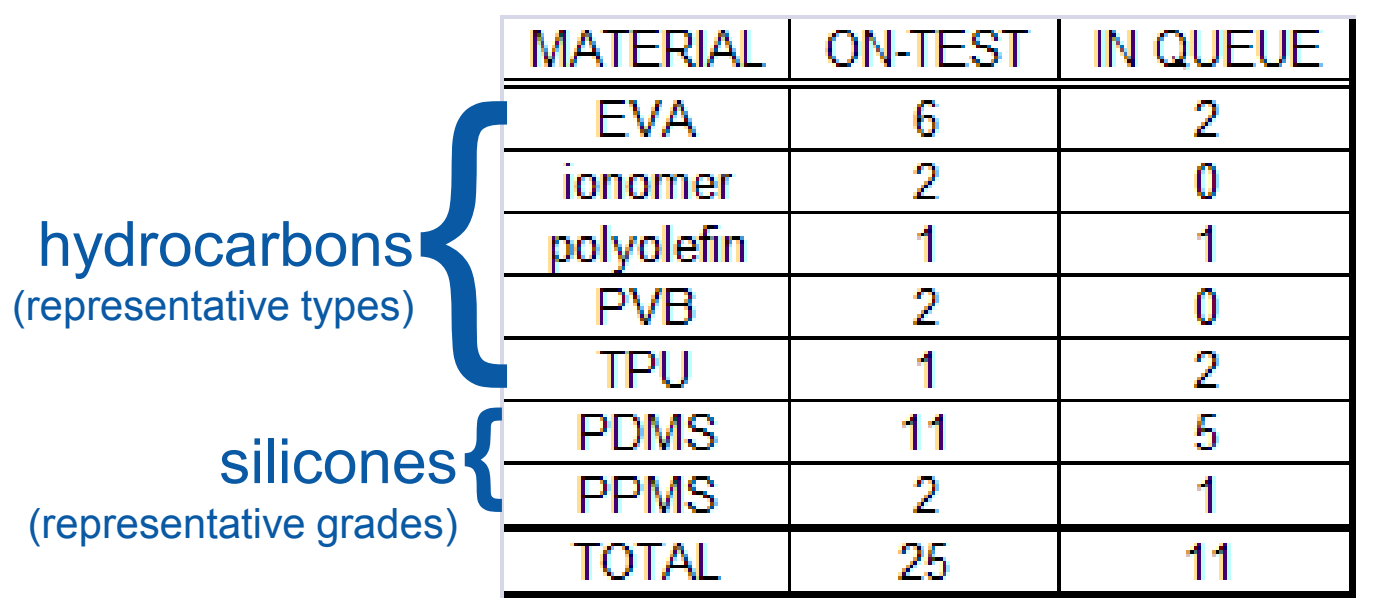

test coupons are mounted in a modified CPV module product on a 2-axis tracker in Golden, CO 


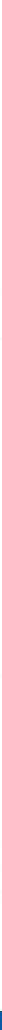


Details of the Experiment (Specimens \& Apparatus)

Miller et. al., PIP, DOI: 10.1002/pip.1241.

hydrocarbons

(representative types)

\begin{tabular}{|c|c|c|}
\hline MATERIAL & ON-TEST & IN QUEUE \\
\hline \hline EVA & 6 & 2 \\
\hline ionomer & 2 & 0 \\
\hline polyolefin & 1 & 1 \\
\hline PVB & 2 & 0 \\
\hline TPU & 1 & 2 \\
\hline PDMS & 11 & 5 \\
\hline PPMS & 2 & 1 \\
\hline TOTAL & 25 & 11 \\
\hline
\end{tabular}

secondary optic (homogenizer)

silicones

(representative grades)

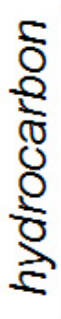
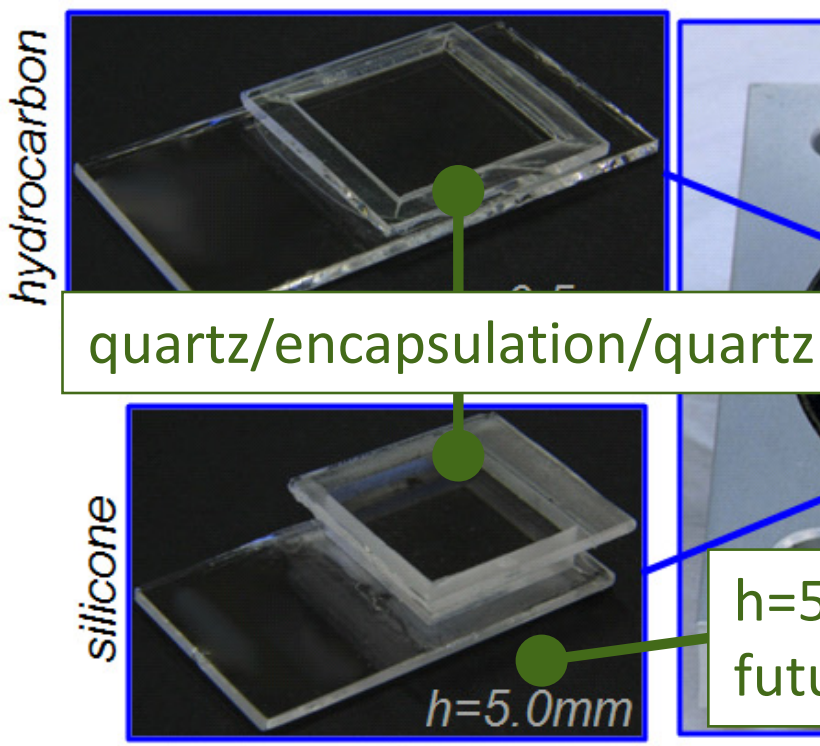

(b)

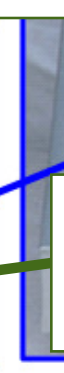

$\mathrm{h}=5 \mathrm{~mm}$ : not advised in future research

(c)
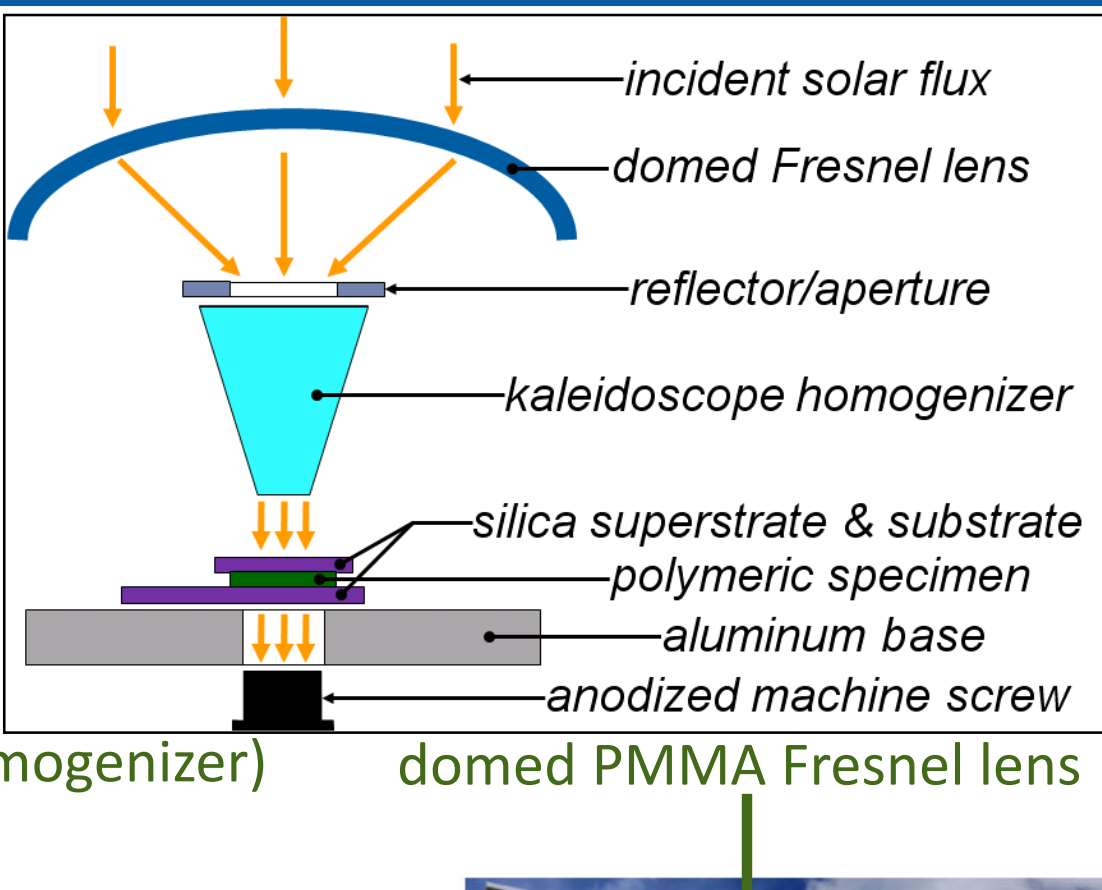

$C_{\mathrm{g}}=500 \mathrm{x}$

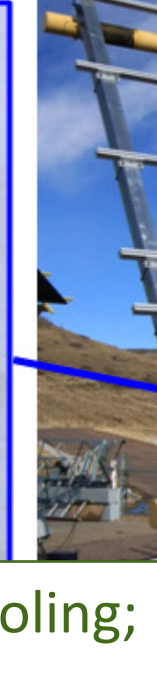

no cell

passive cooling;

$2 \mathrm{~cm}$

(d)

is tracker in Golden, $\mathrm{CO}$ 


\section{Details of the Experiment (Measurands \& Schedule)}

"Continuous" measurements:

ambient conditions (irradiance, temperature, wind...)

fixture temperature (via thermocouple)

Periodic measurements:

$\longrightarrow$ transmittance $(T[\lambda]$, hemispherical \& direct)

mass

appearance (photograph)

$\Rightarrow$ from $T[\lambda]$, calculate: yellowness index (D65 source, $196410^{\circ}$ observer), haze, $\lambda_{\text {cut-on }} \cdots$

$\longrightarrow$ fluorescence spectroscopy

Final measurements:

FTIR, RAMAN, NMR

TGA, DSC (polymer physics)

Test schedule:

$0,1,2,4,6,12,18,24,30,36$... months 


\section{Optical Irradiance May Vary from CPV Transmittance}

-PMMA transmits little

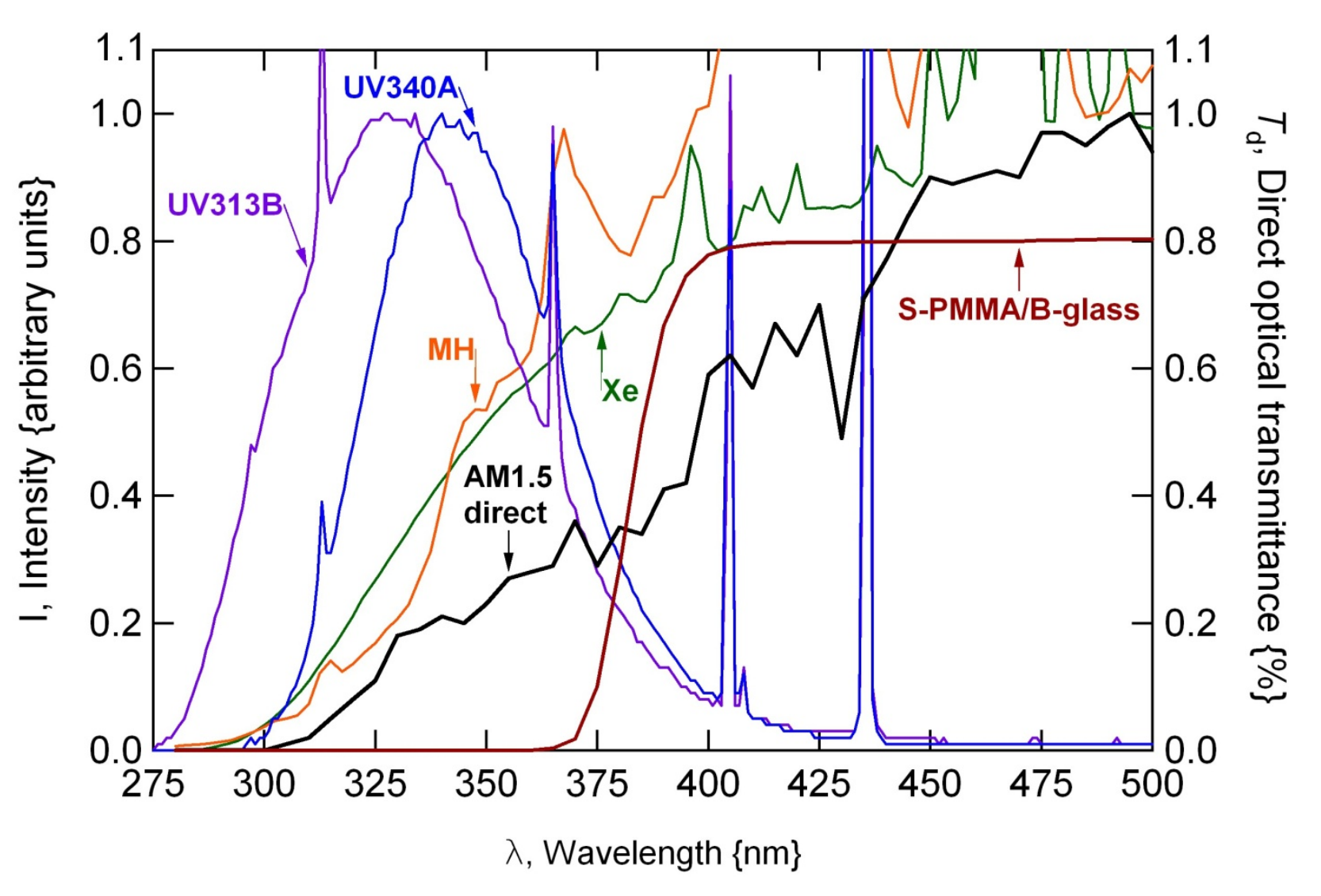

-Thermal content therefore has increased significance (coupled UV \& thermal degradation)

- Some popular indoor sources (UV 313V, UV340A) are completely inappropriate for a PMMA-enabled CPV system

-SoG Fresnel lens is substantially more transmitting ( $T=89 \%$ ) of UV

Miller et. al., PIP, DOI: 10.1002/pip.1241 Irradiance for popular optical sources (including the sun) relative to the CPV optical system 


\section{UV Radiation: Damaging Dose}

-Early weathering studies $\Rightarrow$ total UV dose (damage vs. Joules or hours)

-Activation spectrum instead considers:

1. characteristics of source $\&$ optical system

2. effectiveness of damage at each $\lambda$ ("action spectrum")

3. may be unique to each characteristic (+ and -)

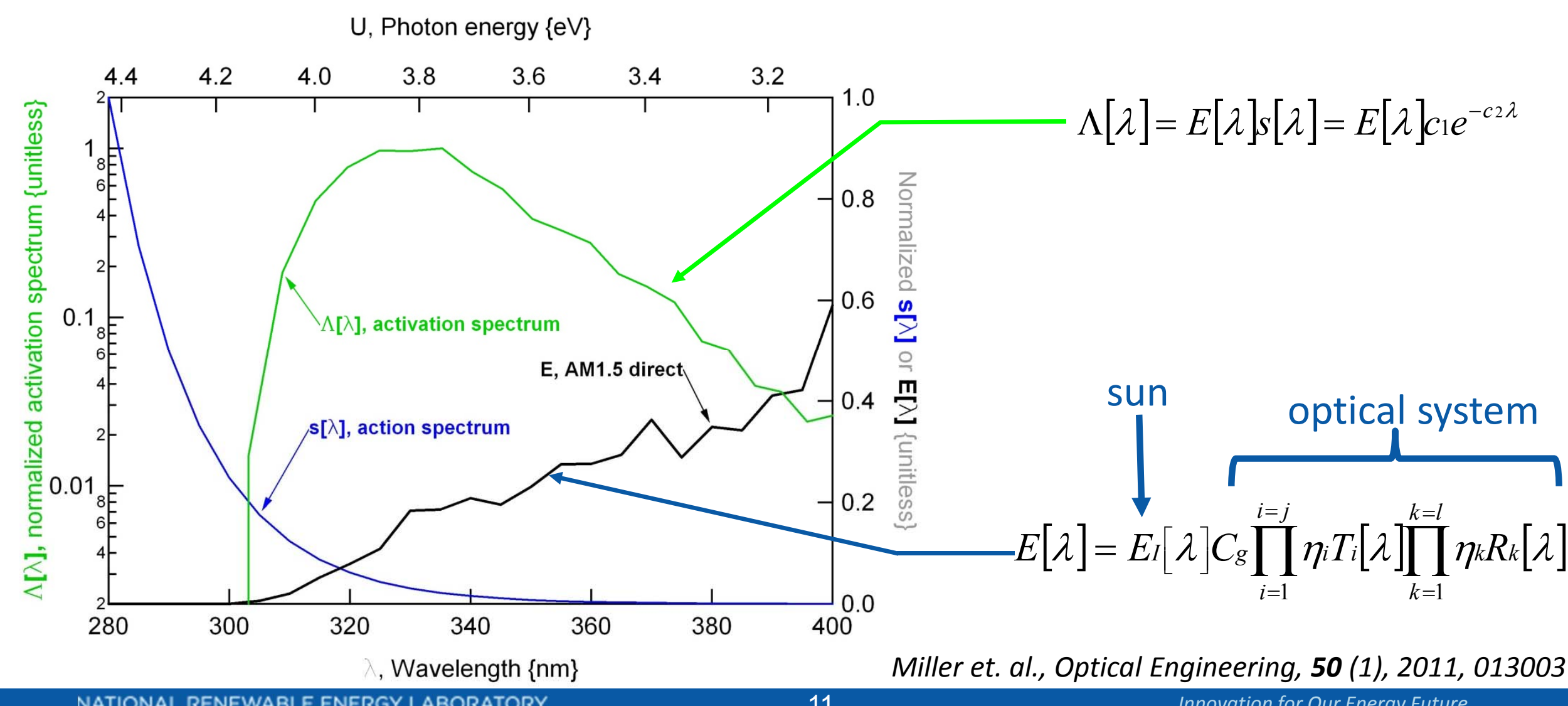




\section{The Optical System Readily Affects UV \& IR Dose}

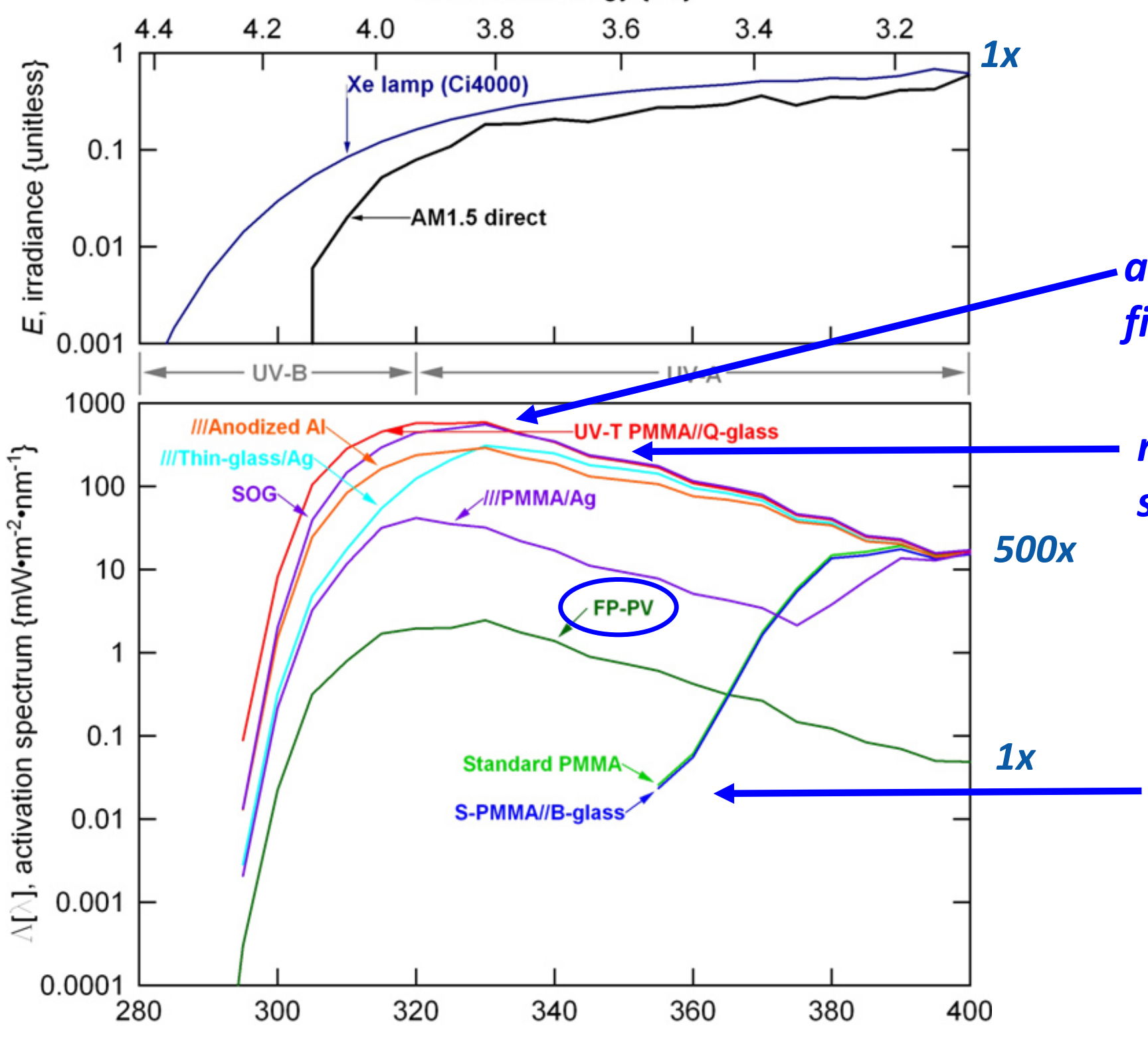

reflective systems or SoG

PMMA refractive systems: reduced UV

$\lambda$, Wavelength $\{\mathrm{nm}\}$

Miller et. al., Optical Engineering, 50 (1), 2011, 013003 


\section{The Field Conditions (Specimen Temperature)}

-Specimen temperature proportional to optical (IR) absorptance (thermal management "system": conduction to the frame.)

-Measured at solar noon. Factors: $T_{\mathrm{amb}}$, irradiance, wind speed - $40^{\circ} \mathrm{C}$ temperature rise observed. $T_{\max } 70-80^{\circ} \mathrm{C}$ in summer.

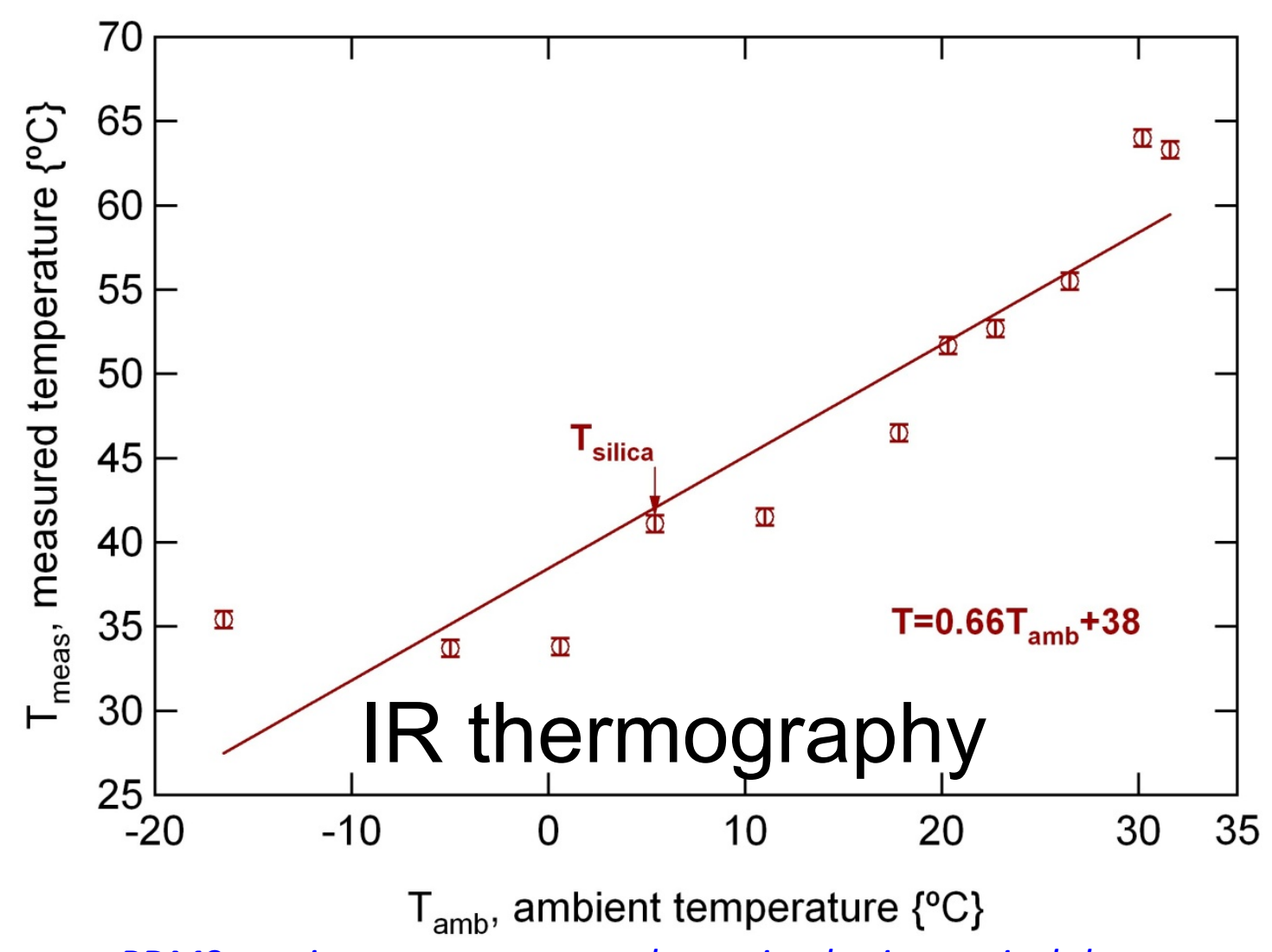

PDMS specimen temperature, determined using optical thermography

Miller et. al. PIP, DOI: 10.1002/pip.1241. 


\section{Thermal Decomposition of the Encapsulation May Occur at High Temperature}

\section{-Thermal stability}

compared using

thermogravimetric analysis

(TGA) @20C.min-1

- Onset of decomposition for hydrocarbons:

$200-300^{\circ} \mathrm{C}$

- Silicones more thermally stable: $T_{\text {onset }} 300-400^{\circ} \mathrm{C}$

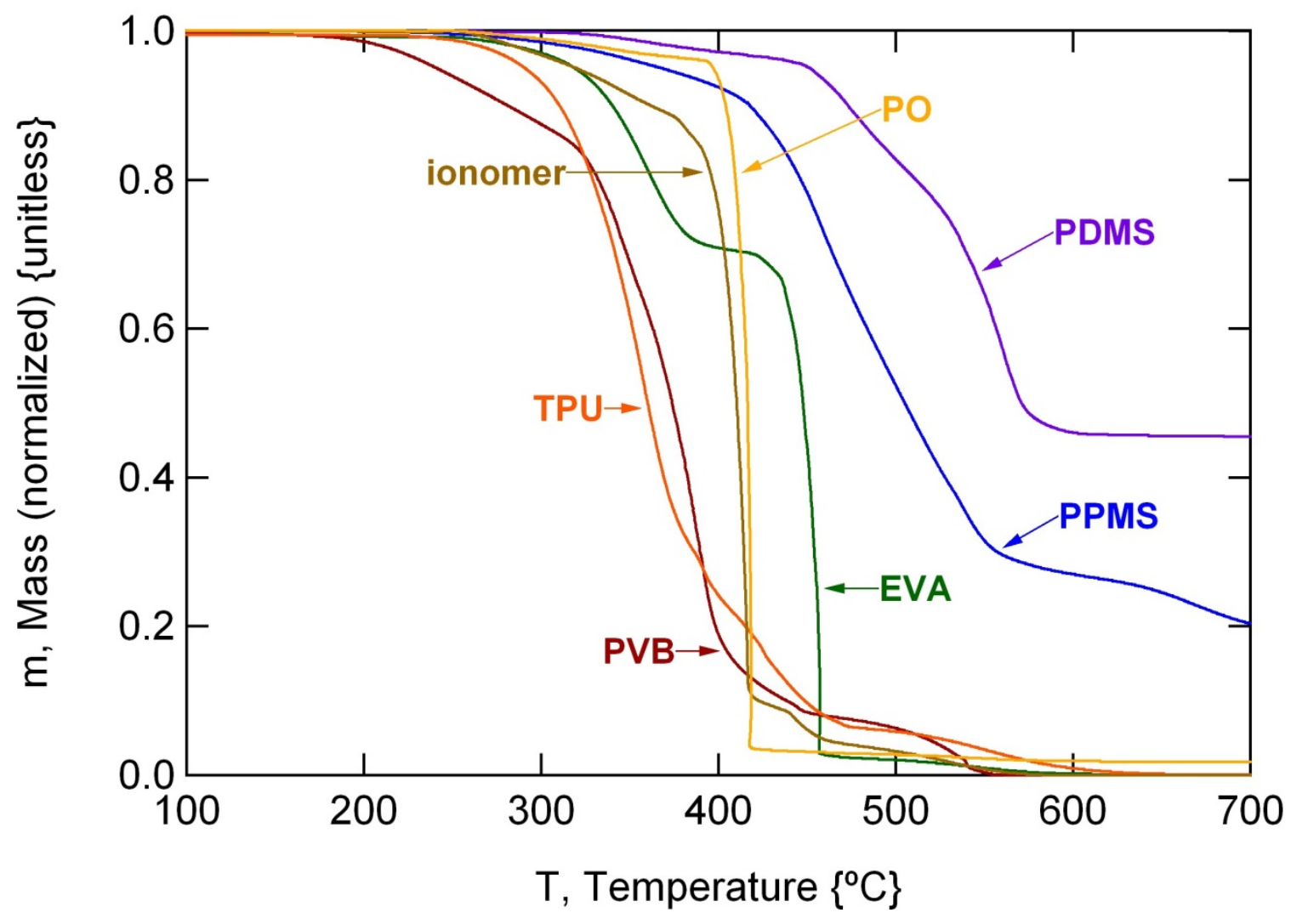

${ }^{*}$ Remember T's for later!

Thermography data for representative materials from the study NATIONAL RENEWABLE ENERGY LABORATORY

Miller et. al., PIP, DOI: 10.1002/pip.1241 


\section{Results of Discovery Experiments}

\section{The Effect of Contamination)}

-Intentionally introduce soil, $\mathrm{Al}, \mathrm{PE}$, or bubbles into EVA or silicone EVA: soil, Al, PE motivated localized discoloration $\Rightarrow$ combustion silicone: soil, $\mathrm{Al} \Rightarrow$ localized cracking. (no primer present) - elapsed time: minutes - days/weeks

- bubbles: no failure @ $C_{\mathrm{g}}=500$, despite 4\% measured $T[\lambda]$ reduction

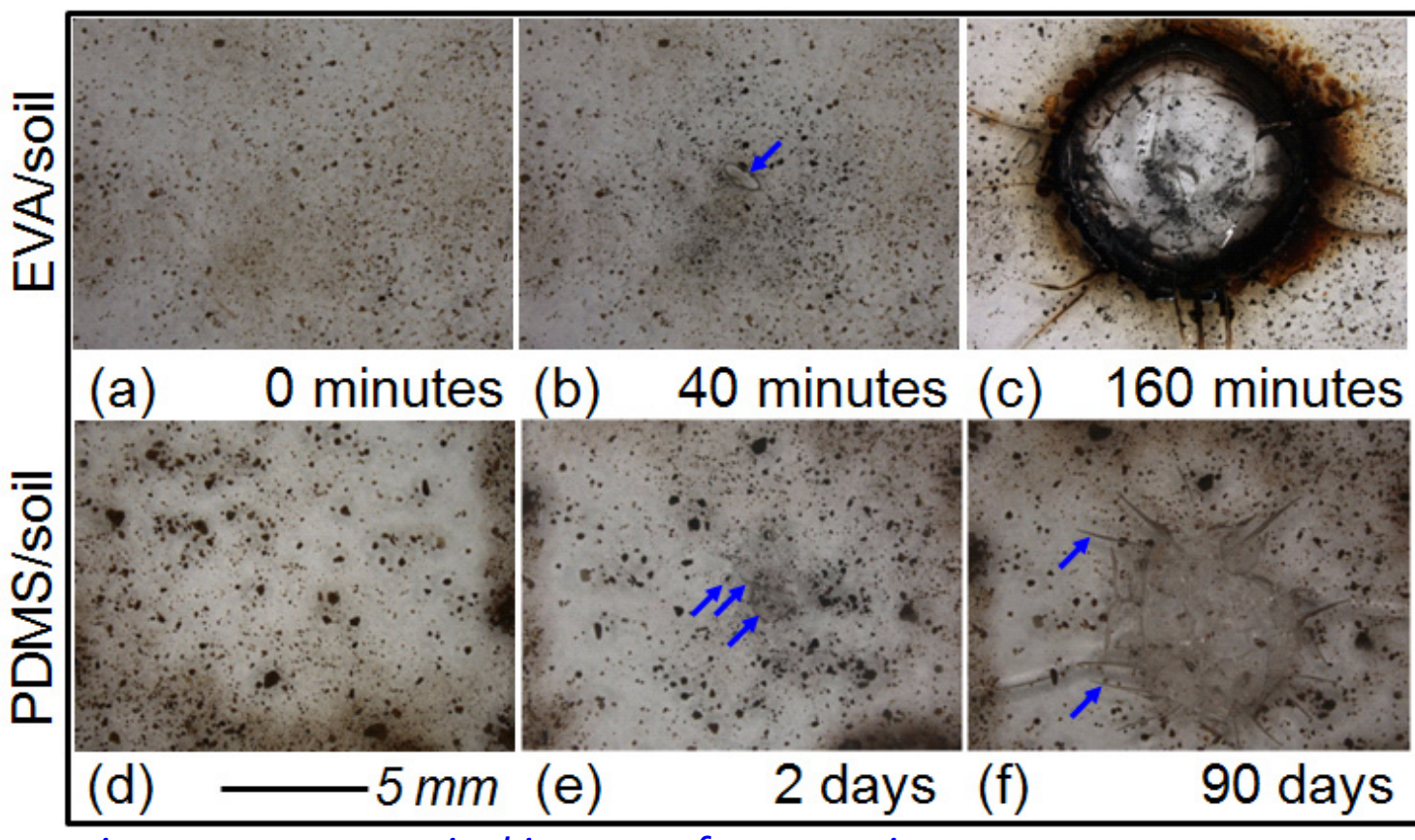

time sequence: optical images of test specimens

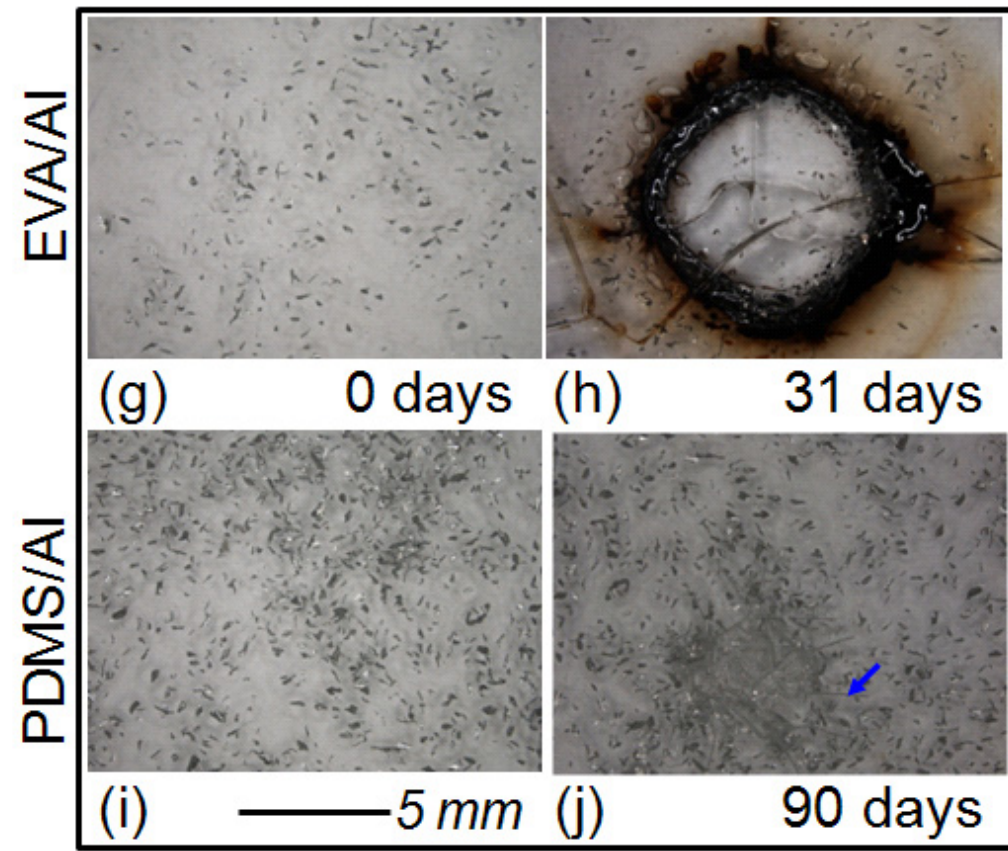

Miller et. al., PIP, DOI: 10.1002/pip.1241 


\section{Results of the Formal Experiment}

\section{(Hydrocarbon Specimens)}

-PVB was the first material to demonstrate thermal runaway mediated failure

-The radius of the affected region was seen to slowly grow during the cold winter months
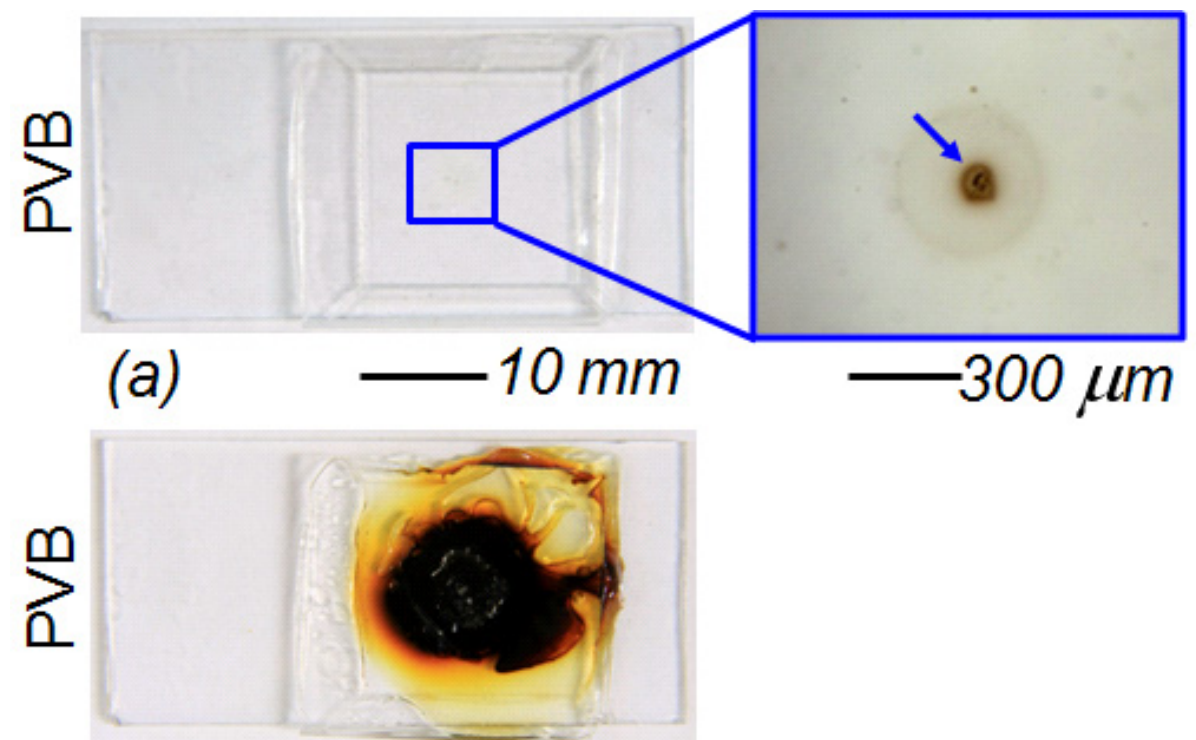

(b) $-10 \mathrm{~mm}$ optical images of test specimen at:

(a) 6 months and (b) 10 months

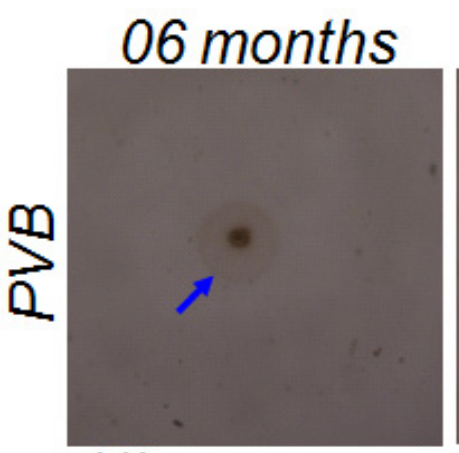

(1)

time sequence: optical images of test specimen
07.5 months

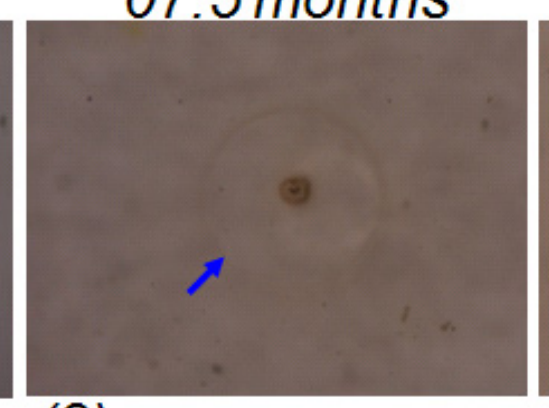

(2)

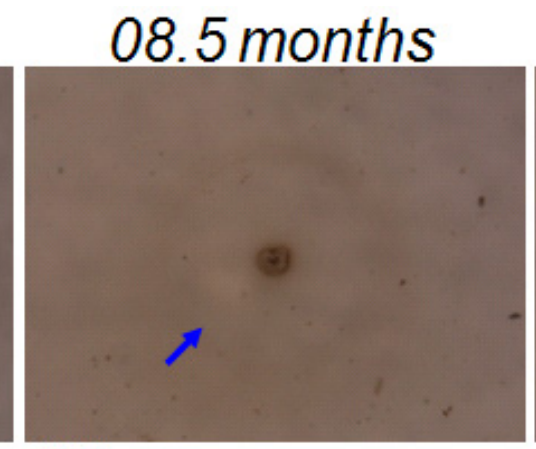

(3)

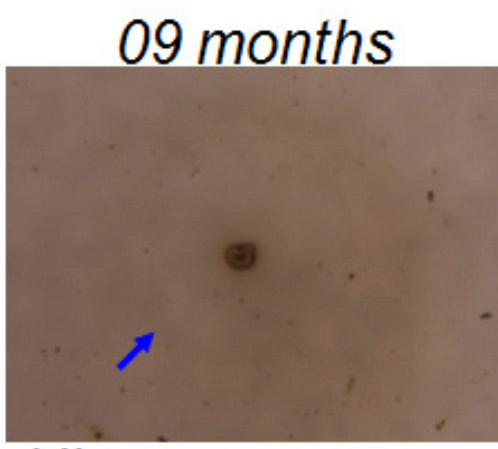

(4)

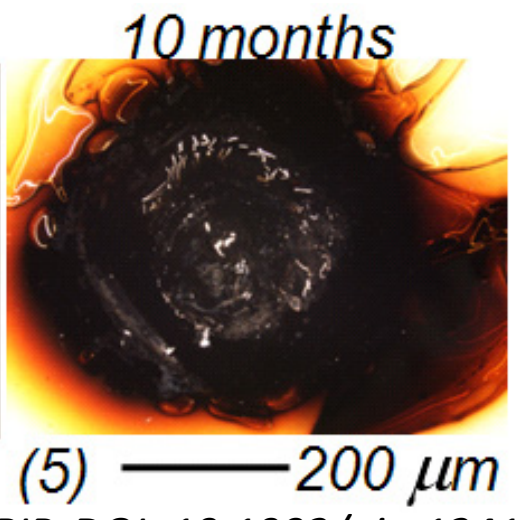

Miller et. al., PIP, DOI: 10.1002/pip.1241 


\section{Results of the Formal Experiment}

\section{(Hydrocarbon Specimens)}

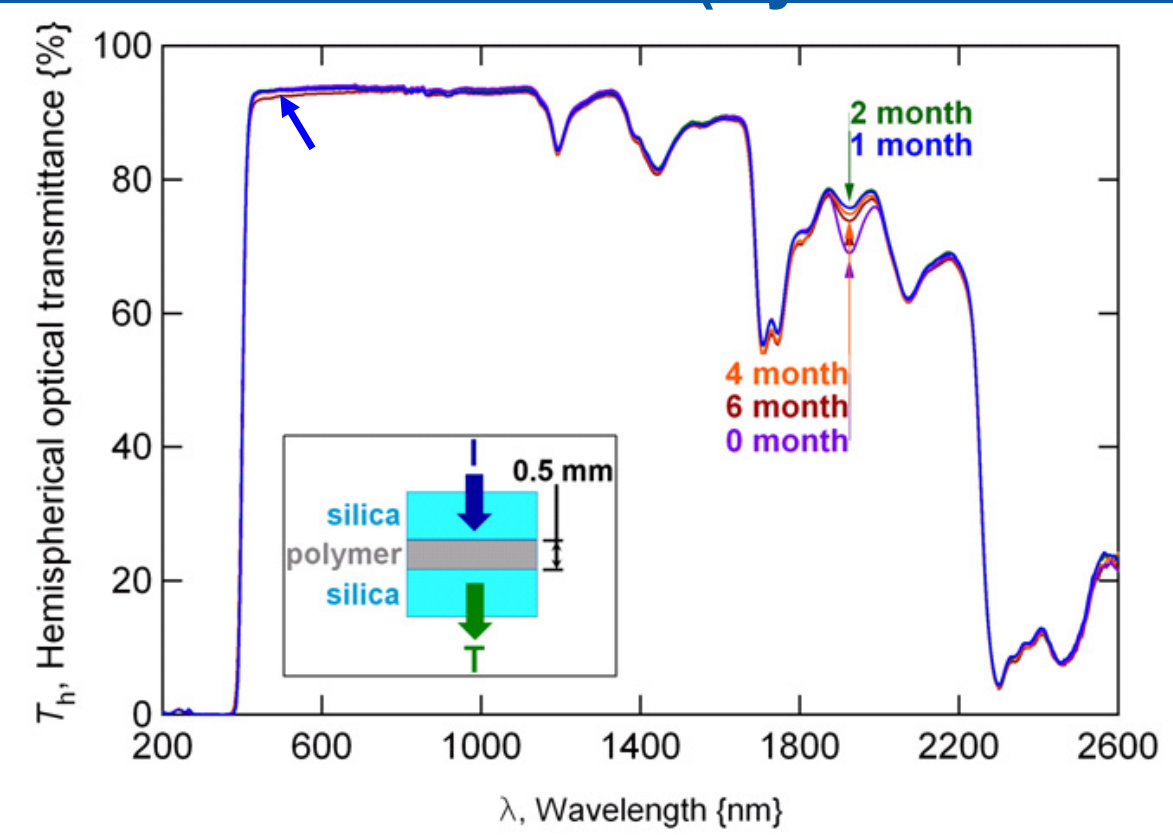

- Transmittance \& YI not significantly affected, despite impending failure

-A diagnostic characteristic with predictive capability is preferred!!!

time sequence: transmittance of the PVB specimen 


\section{Results of the Formal Experiment}

\section{(Hydrocarbon Specimens)}

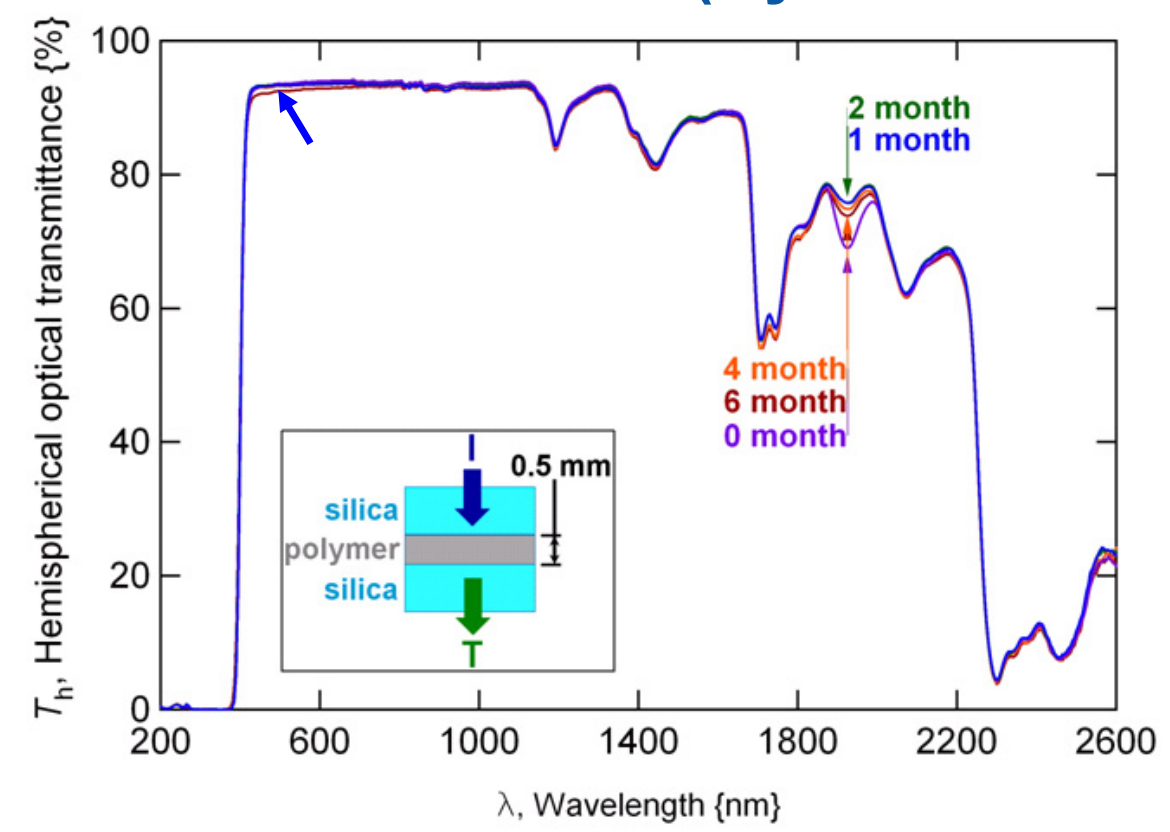

time sequence: transmittance of the PVB specimen

- Optical \& Raman spectroscopy clearly indicate fluorescence

-These techniques may help understand the degradation mechanism (e.g., chromophores)

- Transmittance \& YI not significantly affected, despite impending failure

-A diagnostic characteristic with predictive capability is preferred!!!

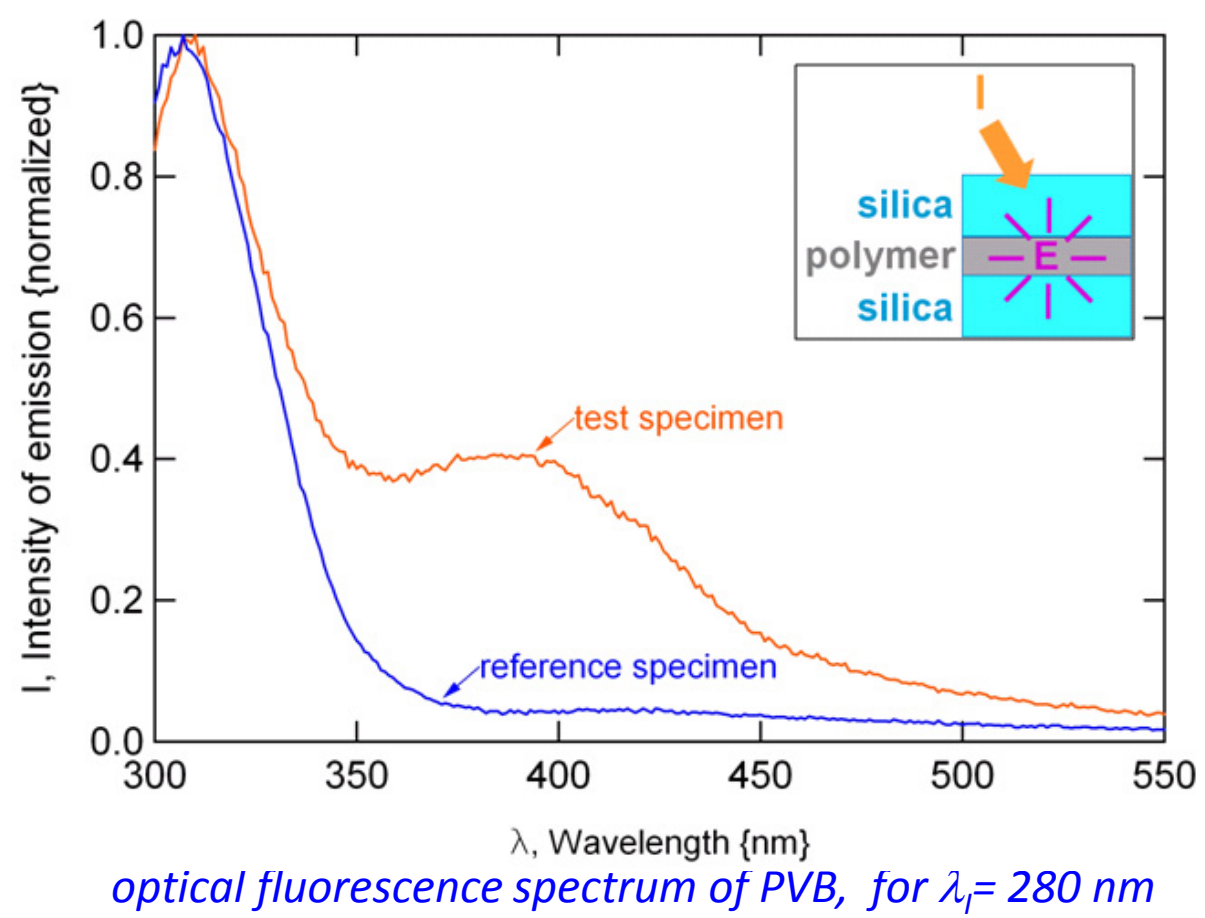




\section{Results of the Formal Experiment}

\section{(Silicone Specimens)}

- Observations of silicone specimens include: (a) densification, (b) cracking, and (c) haze formation

No mass change with time for the (5) densified specimens $\Rightarrow$ likely occurred during molding

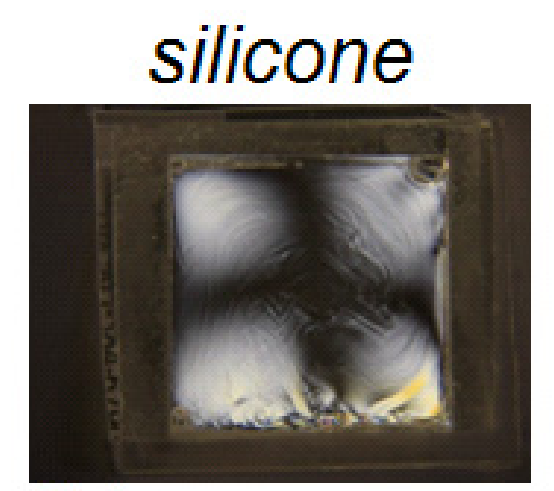

(a)

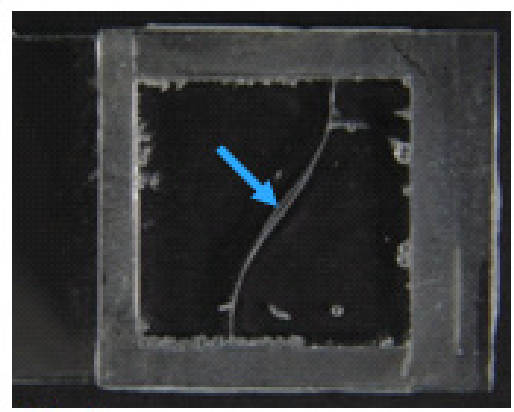

(b)

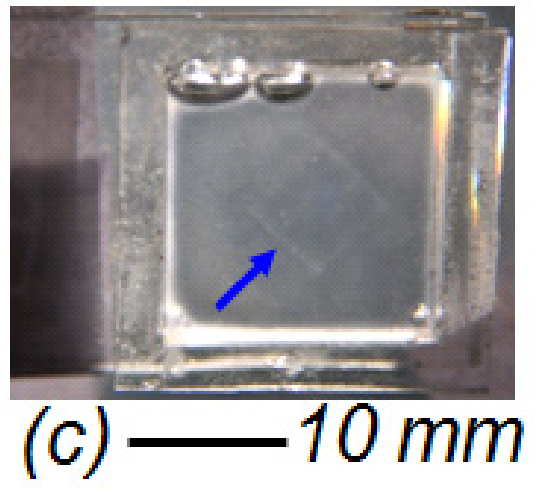

optical images of silicone specimens, including those obtained using (a) cross-polarization or (c) back-lighting 
Results of the Formal Experiment

(Densified Silicone Specimens)

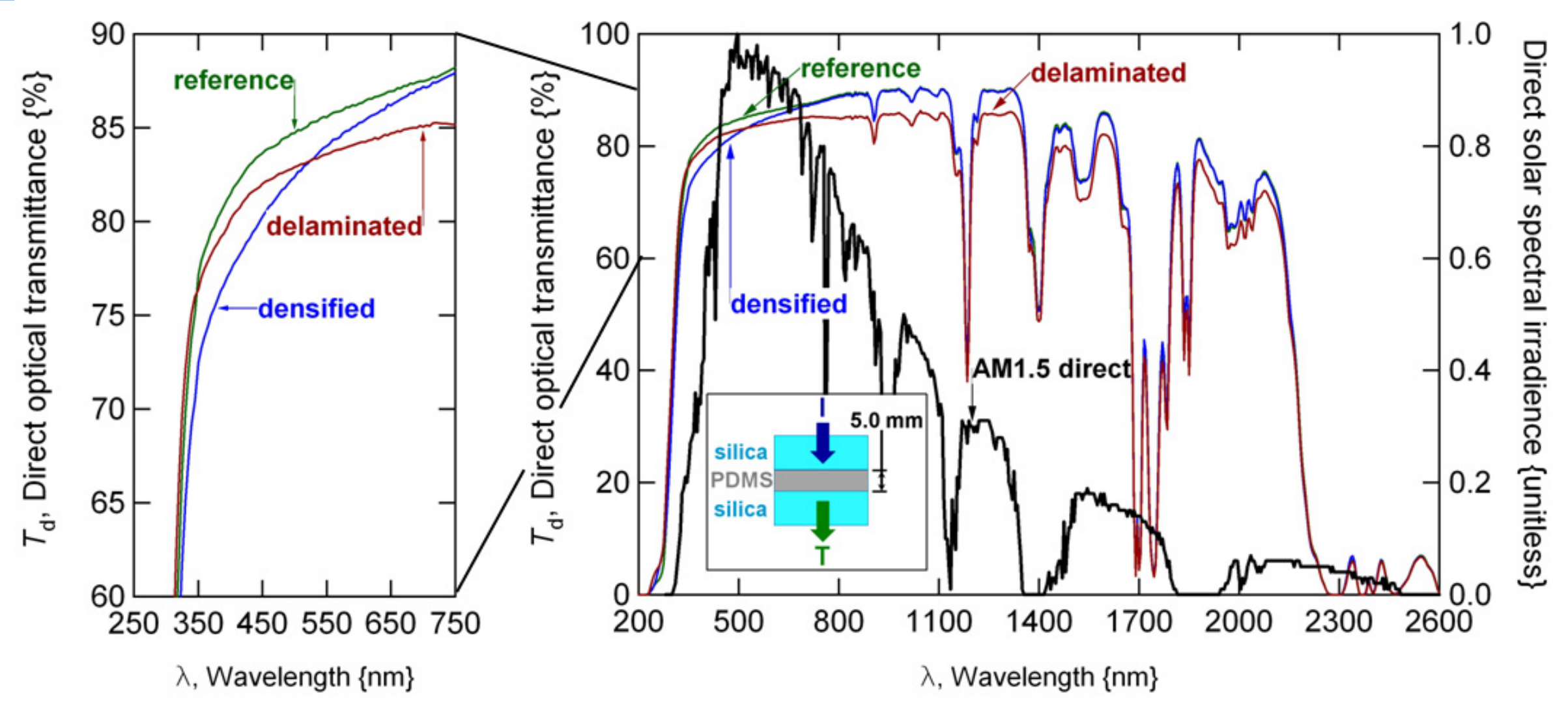

-Densification is not delamination

-Densification does scatter direct light

Problematic for CPV?

-Current limited condition (blue light) $\bullet$ Optical attenuation (less power) $\Rightarrow$ May not be significant in thin bond layers 


\section{Fluorescence Identifies the Silicones Are Affected!}

- Unexpected new peaks identified for all silicone specimens!

-The particular details location and relative intensity of the new $\mathrm{M}_{\mathrm{t}}$ peaks varied with formulation

-Attributed to Pt catalyst (working to verify)

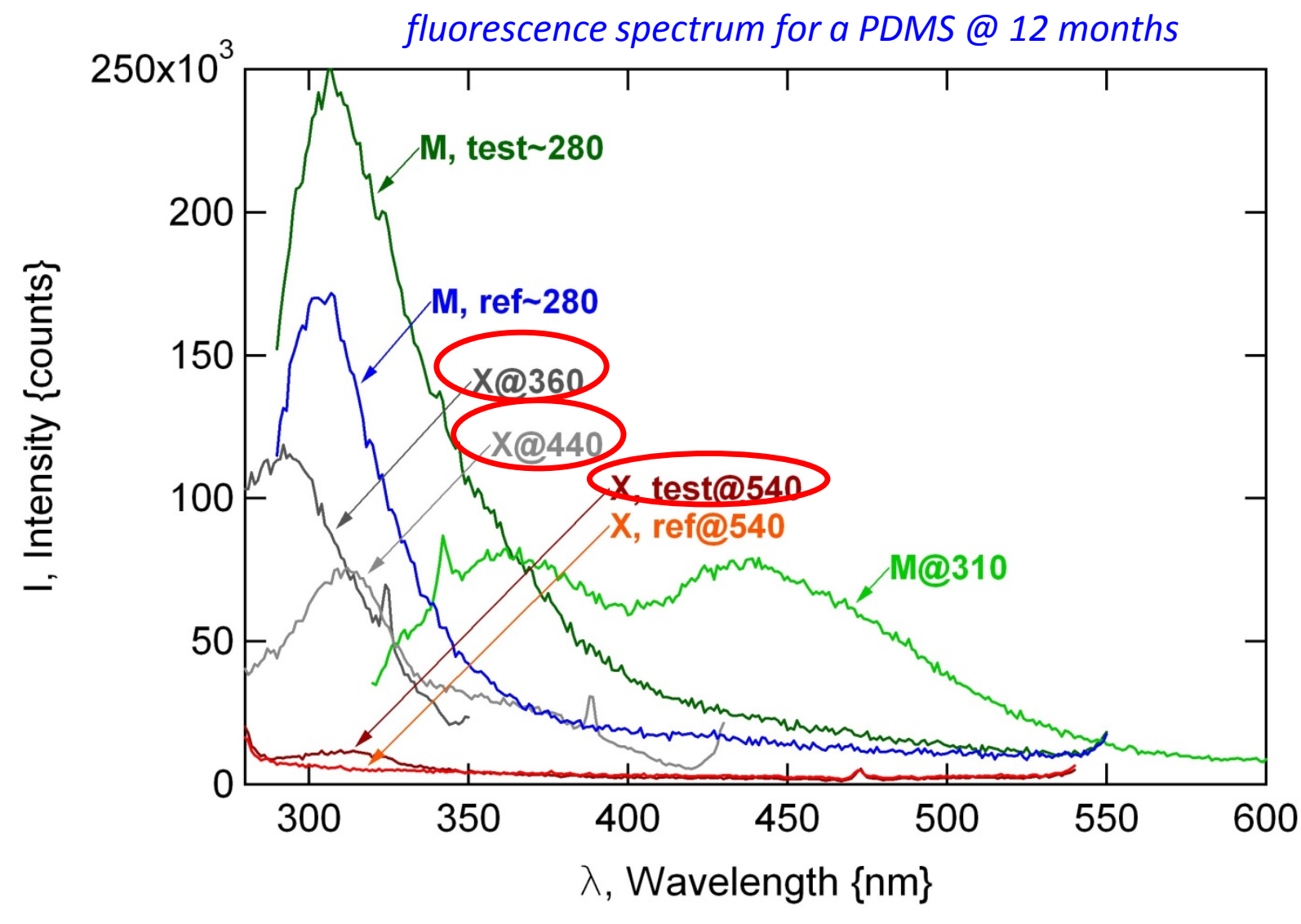

-The implications are unclear. PDMS is historically robust in extreme environments. $\lambda_{X} \ldots<390 \mathrm{~nm}$ for PMMA, $320 \mathrm{~nm}$ for SoG 
- Karstedt's catalyst, Pt(0), examined in tetramethyldivinyldisiloxane

- Catalyst loses fluorescence with UV or T

-Organometallic literature: mononuclear Pt with ligands $\rightarrow$ colloidal Pt, 3-5 nm $\varnothing$

-Discoloration (optical absorptance) could motivate thermal runaway

- No evidence to date of optical degradation in NREL specimens

- Fluorescence of catalyst solution does not correspond to that in $\mathrm{x}$-linked PDMS

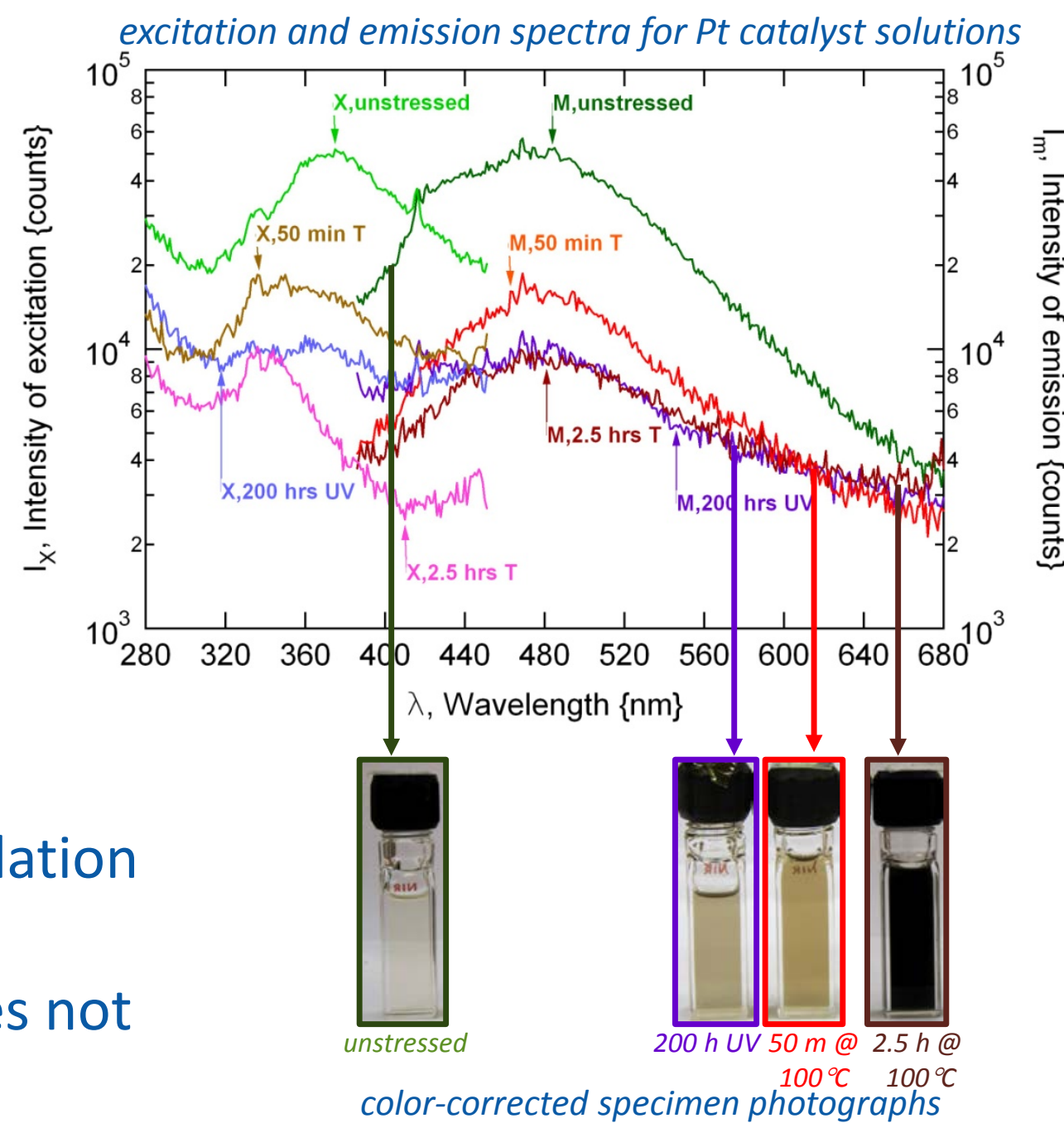

-Alternate pathways: different catalyst type (ligands), peroxide cured silicone, PMMA on glass (PoG) lenses, AR coatings 


\section{UV Can Degrade Silicone Primers}

-Dow-Corning 92-023 used in all NREL PDMS specimens

-The Ti based primer (on glass) reduces

UV transmittance for $\lambda<300 \mathrm{~nm}$

$\left(n \mathrm{TiO}_{2}=2.5\right)$

- Experiments identify primer is quite photoactive:

discoloration with minor fluorescence

-Transparency recovered with time

$\left(\mathrm{O}_{2}\right.$ facilitated?)

transmittance for primer solutions (in IPA)

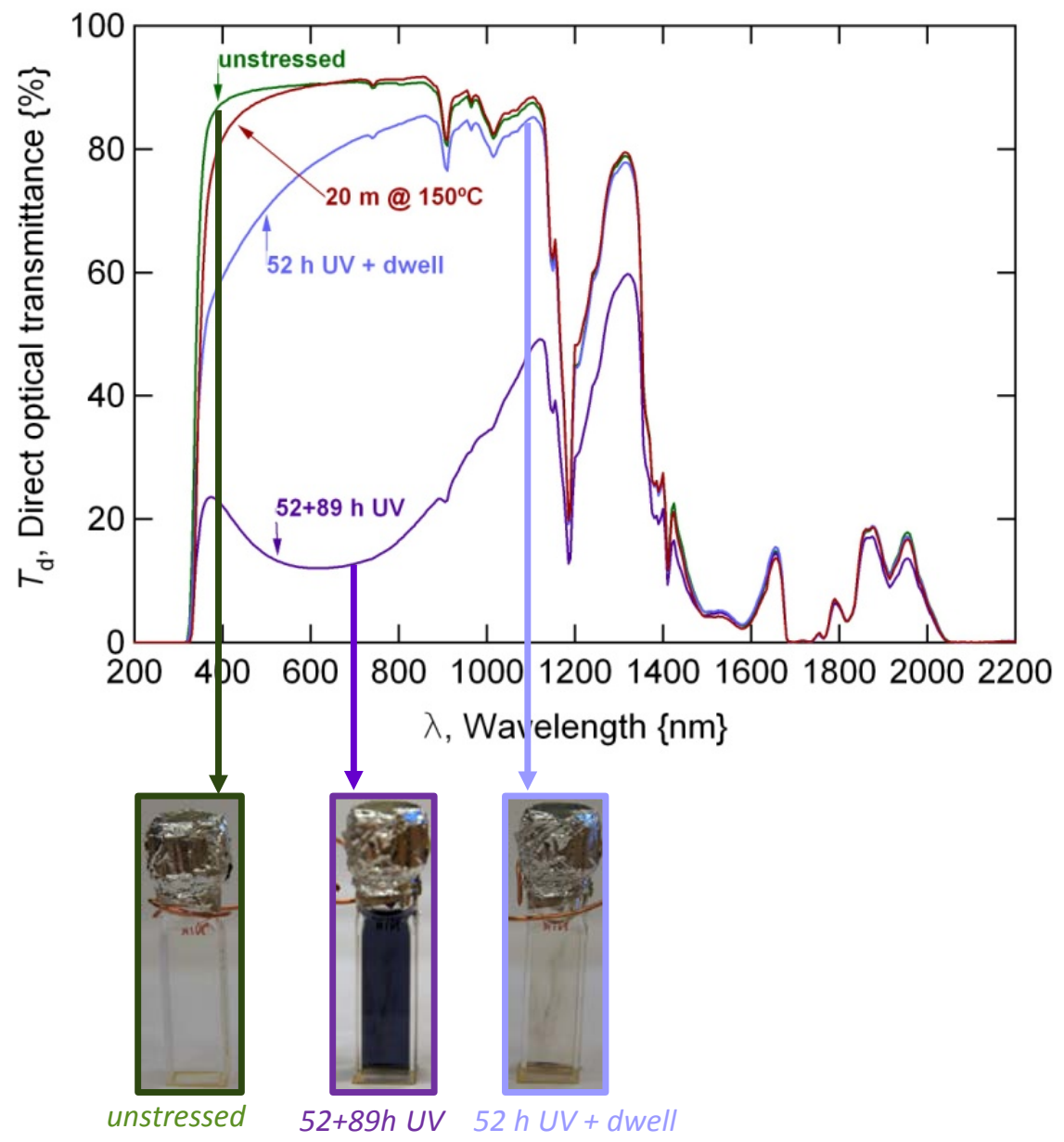

- $\mathrm{TiO}_{2}$ used in self cleaning coatings.

(UV driven consumption of organic contamination). Affect on PDMS is unclear.

-Alternate pathway: $\mathrm{Sn}$ catalyzed primers ( $n \mathrm{SnO}=2.1)$ 


\section{Summary \& Conclusions}

Field study of the durability of polymeric encapsulation materials for CPV

\section{Discovery experiments:}

-Quickly confirmed the importance of an optical homogenizer -Al, soil, polymeric contamination $\Rightarrow T$ runaway \& combustion of EVA -Al, soil contamination $\Rightarrow$ cracking of silicone

\section{Formal experiment:}

-17 of 25 specimens not discussed today! • 3 of 25 specimens "failed". PVB: localized discoloration $\Rightarrow$ thermal runaway $\Rightarrow$ combustion Fluorescence \& Raman spectroscopy may diagnose \& provide prediction Silicone: densification, cracking, haze-formation

Densification affects the direct transmittance

\section{PDMS Fluorescence:}

-Working to understand observed peaks; alternative "solutions" identified *Transmittance of optical system and corresponding activation spectrum of the encapsulation are critical to encapsulation durability 


\section{Acknowledgements}

-NREL: Dr. Keith Emery, Dr. Daryl Myers, Dr. John Pern, Matt Beach, Christa Loux, Tom Moricone, Marc Oddo, Bryan Price, Kent Terwilliger, Robert Tirawat
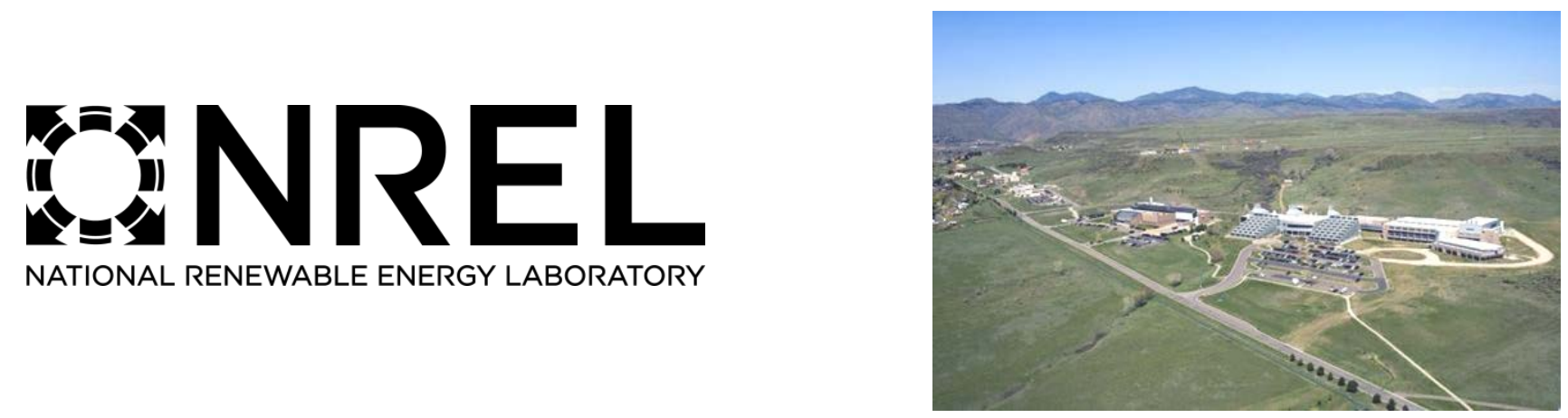

This work was supported by the U.S. Department of Energy under Contract No. DE-AC36-08G028308 with the National Renewable Energy Laboratory.

Paper: "The Durability of Polymeric Encapsulation Materials for Concentrating Photovoltaic Systems", Prog. Photovoltaics, DOI: 10.1002/pip.1241. 